\title{
Repotenciação de um Transformador de Potência Trifásico de 650 kVA / 750 kVA em Proveito do Aumento de Potência
}

\author{
Repowering of a Three-Phase Power Transformer of 650 kVA / 750 kVA in Increase of Power Output
}

\author{
Alex Franco Ferreira*, Guilherme Ferraz de Oliveira
}

Como citar esse artigo. Ferreira, AF; De Oliveira, GF. Repotenciação de um Transformador de Potência Trifásico de $650 \mathrm{kVA} / 750 \mathrm{kVA}$ em Proveito do Aumento de Potência. Revista Teccen. 2018 Jul./Dez.; 11 (2): 40-50.

\author{
Resumo
}

Entre os equipamentos que compõem um sistema de distribuição de energia elétrica, os transformadores são equipamentos essenciais na distribuição de energia, podendo adequar os níveis de tensão do sistema elétrico de potência. Sendo de suma importância a manutenção e por vezes a repotenciação de tais equipamentos. A repotenciação é uma técnica que vem sendo adotada pelas concessionárias e empresas que reusam seus transformadores antigos, visando-se menor custo, melhor prazo de fornecimento e confiabilidade. Este artigo teve por objetivo apresentar as técnicas de aumento de potência nos transformadores antigos das redes de distribuição, aproveitando-se o espaçamento da janela do núcleo e obedecendo-se ao isolamento entre as bobinas. Adotou-se para estudo de caso um transformador de potência abaixador com uma tensão em seu primário de 13.8/11.4kV ligado em Delta, e no secundário uma tensão de 440/254 V ligado em Estrela, com bobinas de cobre eletrolítico, resfriamento com óleo isolante, núcleo com aço silício. Para tornar possível a repotenciação deste transformador seguiram-se as normas vigentes, ABNT NBR 5440:2014 e ABNT NBR 5356:2014, realizando-se os cálculos e ensaios necessários de forma aumentar a potência do transformador para que mesmo atenda com as especificações necessárias do usuário. Concluiu-se que com a aplicação de novas técnicas tem-se uma redução dos impactos ambientais com o descarte dos materiais obsoletos, bem como obteve-se uma redução dos investimentos em um novo transformador. Tornando-se também importante salientar o ganho importante de potência, aproximadamente 100KVA

Palavras-Chave: Repotenciação. Transformador de Potência. Transformador de Distribuição. Ganho de Potência.

\begin{abstract}
Among the equipment that make up an electric power distribution system, transformers are essential equipment in the distribution of energy, and can adjust the voltage levels of the electric power system. Being of paramount importance the maintenance and sometimes the repowering of such equipment. Repowering is a technique that has been adopted by concessionaires and companies that reuse their old transformers, aiming at lower cost, better delivery time and reliability. The aim of this article was to present the power increase techniques in the old transformers of the distribution networks, taking advantage of the spacing of the core window and obeying the insulation between the coils. A lower power transformer with a primary voltage of $13.8 / 11.4 \mathrm{kV}$ connected in Delta was adopted for case study, and in the secondary a voltage of 440/254 V connected in Star, with coils of electrolytic copper, cooling with oil insulating, core with silicon steel. In order to make possible the repowering of this transformer, ABNT NBR 5440: 2014 and ABNT NBR 5356: 2014 were followed, and the necessary calculations and tests were carried out in order to increase the power of the transformer so that it even meets the necessary specifications of the transformer user. The application of new techniques has been reduced to an environmental impact with the disposal of obsolete materials, as well as a reduction of investments in a new transformer. It is also important to have an important gain or gain of power, approximately 100KVA.

Keywords: Repotentiation. Power transformer. Distribution Transformer. Power Gain.
\end{abstract}

\section{Introdução}

Entre os equipamentos que compõem um sistema de distribuição de energia elétrica, os transformadores são equipamentos essenciais na distribuição de energia, podendo adequar os níveis de tensão do sistema elétrico de potência.

Segundo O Setor Elétrico (2009), os transformadores de distribuição representam mais de 2.300.000 unidades em todo o território nacional, destes $57 \%$ são monofásicos e $43 \%$ trifásicos.
De acordo com O Setor Elétrico (2009), existe pesquisa que no território brasileiro exista uma grande quantidade de transformadores de distribuição operando a mais de 25 anos, e que a vida útil de um transformador de distribuição é estimada em 30 anos. Algumas opções surgem para destinação desses equipamentos ultrapassados tecnologicamente.

Atualmente existem diversos estudos para aplicação de métodos como a repotenciação, que vem sendo adotado pelas concessionárias e empresas que reusam seus transformadores antigos, visando menor custo, prazo de fornecimento e confiabilidade. 
O estudo de repotenciação tem uma visão voltada para que o transformador tenha maior vida útil e confiabilidade nas redes de distribuição.

Portanto, o objetivo desse artigo e apresentar as técnicas de aumento de potência nos transformadores antigos, das redes de distribuição, aproveitando-se do espaçamento da janela do núcleo e obedecendo-se ao isolamento entre as bobinas.

Para que se possa repotenciar um transformador é necessário seguir as normas vigentes, ABNT NBR 5440:2014, ABNT NBR 5356:2014, de forma a obterse um equipamento de menor custo onde o mesmo seja capaz de atender com as especificações necessárias do usuário.

O presente artigo apresenta uma metodologia aplicada no reprojeto de um transformador de distribuição, no qual foram aplicadas técnicas de projeto e materiais isolantes com melhores características térmicas e dielétricas.

A sequência metodológica inicia-se com as medições, visando o melhor espaçamento entre a janela do núcleo e as bobinas.

Depois de todo núcleo medido e visto que o mesmo atende a potência desejada realiza-se o primeiro teste de corrente de excitação. Com a realização deste teste executa-se a medição da corrente de excitação que circula pelo núcleo do transformador, sabendo-se que a mesma não pode ultrapassar $1,7 \%$ de acordo com a ABNT NBR 5356:2014, obtém-se assim a quantidade de espiras do secundário.

Concluído o primeiro teste, inicia-se o cálculo do transformador, sendo que a corrente de excitação não deva ultrapassar os limites por norma. Com a quantidade de espiras do secundário, o próximo passo é calcular a bitola do condutor do secundário de acordo com a sua corrente nominal, verificando a bitola do condutor, realizam-se então os devidos cálculos para comprovar-se se os espaçamentos estão corretos no núcleo de acordo com a bobina do secundário, verificado e aprovado o espaçamento calcula-se a quantidade de espira no primário, verificando-se também a bitola do condutor de acordo com a corrente nominal do primário e o espaçamento do núcleo.

Realizados todos os cálculos, inicia-se o enrolamento das bobinas, sendo que a construção vai de acordo com cada projeto, podendo ser inteira ou dividida em panquecas, concluído os enrolamentos realiza-se a montagem do transformador, faz-se então a prensagem e ligação do comutador de tensão.

O primeiro teste a ser realizado após a montagem, é o teste de relação, com esse teste verifica-se a relação de tensão, após o teste o mesmo é direcionado a estufa para realizar secagem de toda a parte ativa, retirandose assim toda umidade e realizando-se a secagem do verniz isolante.

Após a secagem realiza-se o teste de isolação com o aparelho Megger. Com este teste verifica-se se o tempo que o transformador esteve na estufa foi o suficiente para a secagem ou se existe a necessidade de mais algumas horas na estufa. Após realiza-se a montagem do transformador no tanque onde será imerso no óleo isolante. Com o transformador lacrado e imerso no óleo direciona-se o mesmo até o laboratório onde são feitos ensaios determinados na norma, são eles:

$1^{\circ}$ Ensaio a Vazio: Com a finalidade de verificar as perdas no ferro.

$2^{\circ}$ Ensaio de Curto Circuito: Com a finalidade de verificar as perdas no cobre e a impedância do transformador.

$3^{\circ}$ Ensaio de Induzido: Com a finalidade de verificar a isolação entre mínimo uma frequência de $120 \mathrm{~Hz}$.

$4^{\circ}$ Ensaio de Tensão Aplicada: Com a finalidade de verificar se o mesmo está com isolação para suportar a tensão no qual foi calculado.

Após os ensaios gerou-se a nova placa do transformador, afixando-a no mesmo.

\section{Princípio de funcionamento de um transformador}

Para HALLIDAY (2004), ao introduzir um imã em uma bobina a mesma acusa uma presença de corrente elétrica, onde este fenômeno foi caracterizado Lei de Indução de Faraday, onde a intensidade de uma força eletromotriz induzida é igual à variação do fluxo magnético no interior da espira.

Sabe-se que a Lei de Faraday permite calcular o valor da força eletromotriz que é responsável pela corrente induzida no circuito e sua unidade de medida é dada em Ampere.

Segundo Santos (2016), o campo magnético induzido apresenta uma reação contrária da ação provocada pelo imã, quando o norte do imã se aproxima da espira, o sentido da força eletromotriz é anti-horário. Isso ocorre porque o Norte será o sentido positivo da indução magnética, que com isso o movimento das cargas positivas se coincide com o sentido da força eletromotriz induzida. Para que possa mudar o movimento de uma carga que está no condutor, a carga deverá receber um impulso de uma força que será aplicada

De acordo com Almeida (2000), o funcionamento do transformador é baseado nas leis de Faraday e Lenz, sendo um dispositivo de corrente elétrica alternada que opera nos princípios do magnetismo, onde será destinado na alteração dos parâmetros físicos de tensões, correntes e impedâncias.

Para Oliveira e Ferreira (2016), a composição do transformador será simples consistindo em 2 bobinas de um material condutor, constituído por uma bobina no primário que recebe a tensão e a corrente que lhe foi 
dimensionada, e uma bobina no secundário que recebe esta tensão e corrente sendo transferida a uma carga, a tensão que se deseja obter no secundário será relativa a quantidade de espiras que compõe o primário, onde terá um circuito magnético onde essas bobinas serão acopladas, onde o condutor magnético é constituído de um núcleo de material ferromagnético, com a finalidade de produzir um caminho de baixa relutância para o fluxo magnético gerado.

O núcleo dos transformadores será sempre laminado para reduzir a indução de suas correntes parasitas (corrente de Foucault), que serão produzidas no próprio núcleo. Onde as mesmas contribuem para o surgimento de perdas por aquecimento, devido ao efeito Joule, por isso o núcleo de hoje é o de ferro silício com a finalidade de aumentar essa resistividade e diminuir ainda mais as correntes parasitas.

Transformadores são dispositivos elétricos com a finalidade de isolar, elevar ou abaixar uma tensão servem como filtros nos sistemas de potência de transmissão e distribuição de energia (WEG, 2016).

Existem vários tipos de transformadores, cada um para sua função e com potências específicas, sendo que o transformador alvo de estudo neste artigo foi o transformador de distribuição.

\section{Transformador de distribuição}

Segundo Lorencini (2016), transformador de pequeno porte na utilização de rebaixar as tensões recebidas das linhas de distribuição para a alimentação dos consumidores finais, sendo dotado de apenas dois enrolamentos, um de alta tensão e outro em baixa tensão, com comutação a vazio do lado da alta tensão. Encontram-se com os níveis de tensão no primário de até $34.5 \mathrm{kV}$ e no secundário de até 440 volts.

\section{Normas utilizadas no projeto de um transformador}

Utilizam-se normas como orientação na fabricação do transformador, desde os cálculos dos enrolamentos até os ensaios finais, sendo elas as normas ABNT NBR 5440: 2014, ABNT NBR 5356: 2014.

\section{ABNT NBR 5440:2014 - transformadores para redes de distribuição}

A ABNT NBR 5440:2014, orienta na parte de ensaios dos transformadores, desde o primeiro ensaio realizado após a montagem da parte ativa do transformador, até os ensaios mais específicos realizados em laboratórios, realiza-se os seguintes ensaios, onde não se pode ultrapassar valores estabelecidos pela norma, sendo eles:

- Medição de relação de transformação.

- Medição de resistência de isolamento.

- Ensaios a vazio.

- Ensaio de curto circuito.

\section{ABNT NBR 5356:2014 - transformadores para redes de distribuição}

A ABNT NBR 5356:2014, orienta na parte de montagem do transformador, corrente nominal que cada enrolamento deverá possuir, orienta nos enrolamentos do primário e secundário do transformador, os tipos de ligação dos enrolamentos, sobre os espaçamentos entre as bobinas e também espaçamento entre o tanque do transformador, informando também como realizar um fechamento correto do transformador.

\section{Bobinas}

Conforme suas características elétricas, mecânicas e custo, as bobinas são a parte mais importante de um transformador, eletricamente devem conter isolamento da sua classe de tensão e temperatura de operação compatível com sua classe de temperatura. Mecanicamente a bobina estará sujeita a esforços derivados das forças eletromagnéticas, durante os curtos-circuitos nas linhas que o alimenta, e no mais uma parcela bem significativa de custo está no enrolamento do transformador, a bobina deve levar em consideração diversos fatores, tais como:

- A distribuição da tensão ao longo do enrolamento;

- As perdas adicionais;

- Disposição geométrica para facilitar a montagem, construção e ligações;

- E custo na fabricação.

\section{Estudo de caso: Repotenciação de um Transformador}

O transformador escolhido foi um transformador de potência de $650 \mathrm{kVA}$, sendo ele abaixador com entrada em (delta) de 13,8/11,4 kV e saída em (estrela) de 440/254 volts, com 5 Tap's de derivações, e imerso a óleo isolante mineral.

Dados de Placa do Transformador:

- Potência- 650 kVA;

- Frequência- $60 \mathrm{~Hz}$;

- Fases- 3;

- Data de Fabricação- 06/1998;

- Peso Total- $2620 \mathrm{Kg}$;

- Líquido Isolante- 650L;

- Impedância- 4,58\% em 13.8kV; 

Estrela;

- Ligação do primário em $13.8 \mathrm{kV}$ em Delta;

- Ligação do secundário em 440/254 volts em

- Comutador de derivações do primário- 1380013200-12600-12000-11400 volts;

As Figuras 1 e 2 apresentam o transformador danificado já desmontado em seu recebimento para

Figura 1. Transformador danificado.

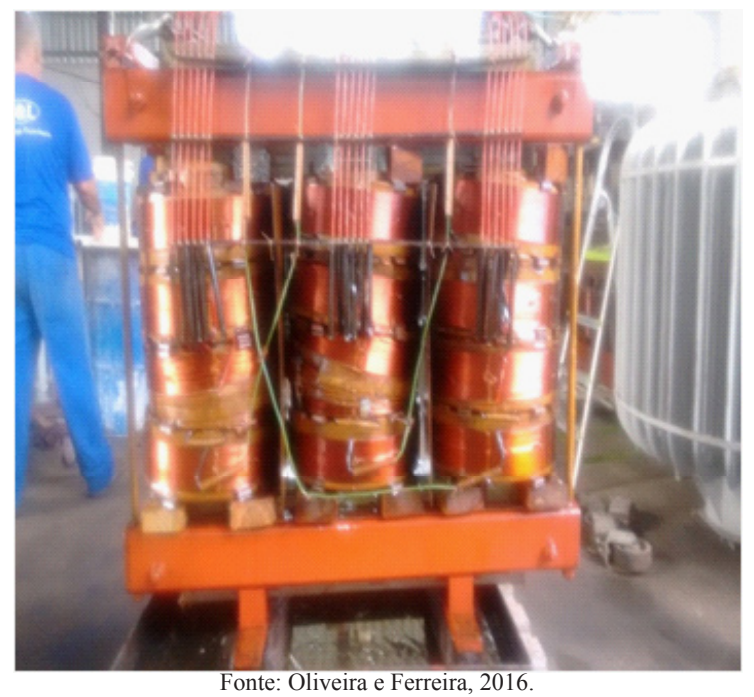

Figura 2. Comutador de derivação danificado.

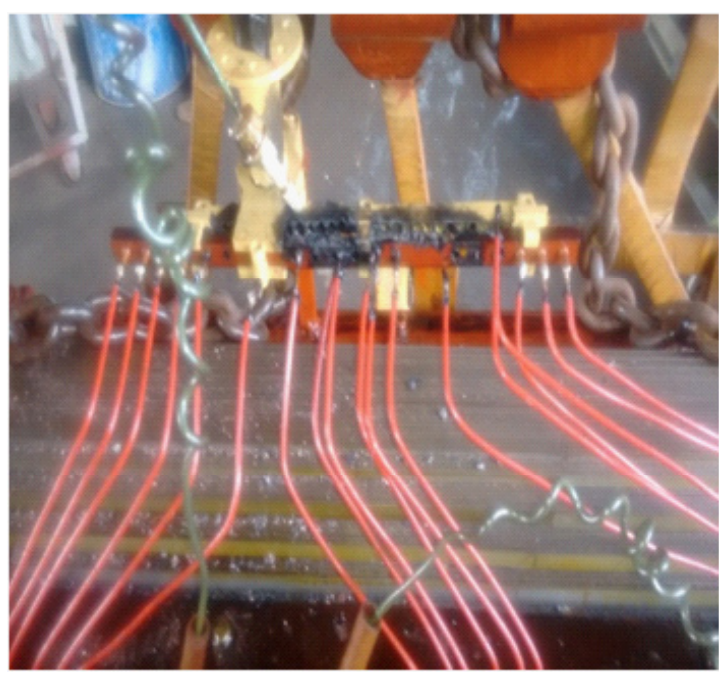

Fonte: Oliveira e Ferreira, 2016.

manutenção.

Não foi possível realizar nenhum ensaio inicial, pois o transformador teve suas bobinas danificadas e sem quaisquer condições de teste.

\section{Núcleo do transformador escolhido}

O núcleo utilizado foi um núcleo de ferro com banho em aço silício e com seu corte em $45^{\circ}$, foram realizados testes necessários para achar o valor da corrente de excitação (a norma orienta que a corrente não poderá ultrapassar $1,7 \%$, passando desse valor a perda a vazio e a perda total de seu transformador será grande, causando prejuízo em valores ao seu projeto).

O valor encontrado para o projeto foi de $1,6 \%$ totalizando 20 espiras no seu secundário.

Projeto de Repotenciação

O projeto apresentado tratou de uma análise técnica de um transformador, contendo sua construção e ensaios elétricos conforme as normas vigentes da ABNT, com as seguintes características:

- Potência nominal: $750 \mathrm{kVA}$;

- Tensão primária: $13.8 \mathrm{kV}$;

- Tensão secundária: 440/254 volts;

- Grupo de ligação: delta-estrela aterrado;

- Números de derivações: 5 (13.8 - 13.2 - 12.6 $12.0-11.4 \mathrm{kV})$

\section{Bobina de Baixa Tensão (Cálculo)}

A bobina de baixa tensão fora definida como helicoidal, conforme Figuras 4 e 5 (mais utilizada em transformadores de distribuição), contendo 20 espiras cada coluna, fora encontrado esse valor de espiras devido ao ensaio realizado no núcleo.

Segundo Martignoni (1969), o cálculo da bobina de baixa tensão pode ser obtido através da Equação 1.

$$
I_{\sec }=\frac{s}{\sqrt{3} \cdot v_{1}}
$$

Onde:

$\mathrm{S}=$ Potência nominal do transformador

$\mathrm{V}_{1}=$ Tensão de fase no secundário do transformador $(440 \mathrm{~V})$

$\mathrm{I}_{\mathrm{sec}}=$ Corrente no secundário

$$
I_{\text {sec }}=\frac{750000}{\sqrt{3} \cdot 440}=984 A
$$

Ainda de acordo com Martignoni (1969), obtémse a seguinte bitola do condutor da baixa tensão com a Equação 2:

$$
\mathrm{d}_{\text {cond }}=\frac{\mathrm{I}_{\text {sec }}}{\text { densidade }}
$$

Onde:

$\mathrm{d}_{\text {cond }}=$ Bitola do condutor BT

$\mathrm{I}_{\mathrm{sec}}=$ Corrente no secundário

$$
\mathrm{d}_{\text {cond }}=\frac{984}{3,3}=298 \mathrm{~mm}^{2}
$$

Definiu-se que a bobina deveria ser construída em duas camadas, adotando-se o sentido para a direita, sendo 10 espiras por camada e serão construídas 3 
Figura 3. Núcleo escolhido.

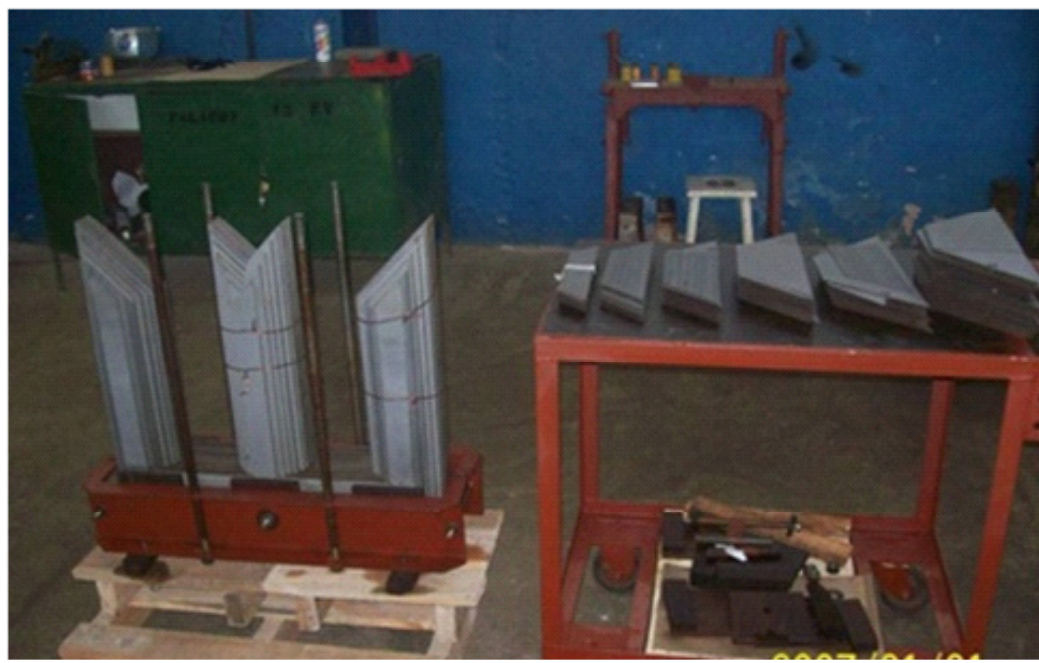

Fonte: Oliveira e Ferreira, 2016

Figura 4. Bobina Helicoidal.

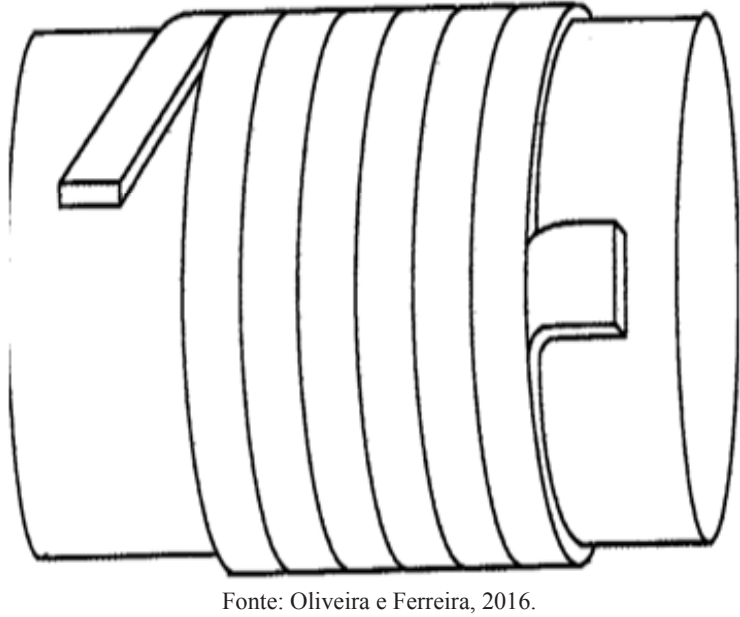

bobinas, pois, o transformador é trifásico, sendo uma bobina por fase.

Condutor nu $\left(\mathrm{mm}^{2}\right)=11$ x $3 \mathrm{~mm}$

Para a seção total do condutor atingir a bitola necessária calculada pela densidade, observa-se que serão necessários 9 condutores por espira, pois:

$11 \times 3=33 \mathrm{~mm}^{2} \times 9=297 \mathrm{~mm}^{2}$

\section{Bobina de Média Tensão (Cálculo)}

A bobina de média tensão fora adotada discoidal, sendo feita em panquecas, separadas por calços e com espaçamento evitando curto circuito entre uma bobina e outra. Foi escolhido esse tipo de bobina com a finalidade de melhorar a refrigeração do transformador.

Segundo Martignoni (1969), o cálculo da bobina de alta tensão pode ser obtido através da Equação 3.
Figura 5. Bobina Helicoidal Finalizada

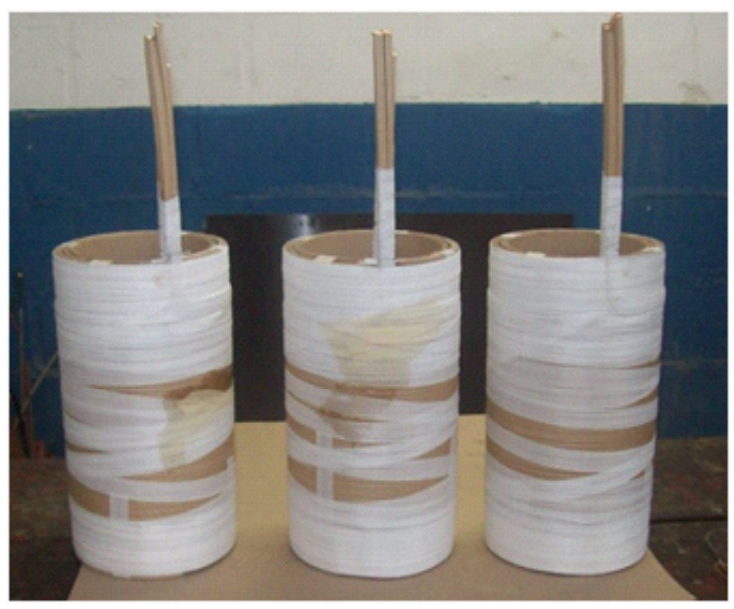

Fonte: Oliveira e Ferreira, 2016.

$$
\mathrm{Ip}=\frac{\mathrm{Pn}}{\mathrm{Vp} \cdot \sqrt{3}}
$$

Onde:

$\mathrm{Ip}=$ Corrente do primário

$\mathrm{Pn}=$ Potência nominal $(750 \mathrm{Kva})$

$\mathrm{Vp}=$ Tensão do primário (13800volts)

$$
I p=\frac{750000}{13800 \cdot \sqrt{3}}=31.37 \mathrm{~A}
$$

Martignoni (1969) afirma que se sabendo a corrente do primário, obtém-se a bitola em $\mathrm{mm}^{2}$ do condutor através da Equação 4.

$$
I p=\frac{31,37}{\sqrt{3}}=18,11 / 3,1=5,84 \mathrm{~mm}^{2}
$$


Definido a bitola do primário, realizou-se a conversão de $\mathrm{mm}^{2}$ para AWG através do Quadro 1, através do quadro observa-se que se deve utilizar o condutor 10 AWG.

Para definir o número de espiras, calcula-se o volt por espira.

Segundo Martignoni (1969), o cálculo tensão por número de espiras pode ser obtido através da Equação 5.

$$
\mathrm{V} / \text { espiras }=\frac{\mathrm{vs}}{\text { Nespiras }}
$$

Onde:

V/esp. $=$ Volt por espiras

Vs $=$ Tensão do secundário (254volts)

Nesp. $=$ Número de espiras (20)

$$
\mathrm{V} / \text { espiras }=\frac{254}{20}=12,7 \mathrm{~V}
$$

De acordo com Martignoni (1969), após calcular o volt por espira, calcula-se a quantidade de espira por cada coluna, e depois divide-se pela quantidade de panquecas, através da Equação 6.

$$
\text { Espira/total }=\frac{\mathrm{Tp}}{\text { Volts espiras }}
$$

Espira/total $=$ Total de espira

$\mathrm{Tp}=$ Tensão primária (13800volts)

Volts espiras $=$ Volts por espira $(12.7)$

Obtendo-se então:

Quadro 1. Conversão de bitolas AWG para mm2.

\begin{tabular}{|c|c|c|}
\hline Número AWG & Diâmetro (mm) & Secção $\left(\mathbf{m m}^{\mathbf{2}}\right)$ \\
\hline 0000 & 11,86 & 107,2 \\
\hline 000 & 10,40 & 85,3 \\
\hline 00 & 9,226 & 67,43 \\
\hline 0 & 8,252 & 53,48 \\
\hline 1 & 7,348 & 42,41 \\
\hline 2 & 6,544 & 33,63 \\
\hline 3 & 5,827 & 26,67 \\
\hline 4 & 5,189 & 21,15 \\
\hline 5 & 4,621 & 16,77 \\
\hline 6 & 4,115 & 13,30 \\
\hline 7 & 3,665 & 10,55 \\
\hline 8 & 3,264 & 8,36 \\
\hline 9 & 2,906 & 6,63 \\
\hline 10 & 2,588 & 5,26 \\
\hline
\end{tabular}

Fonte: Milimétrica, 2016.

\section{Construção do transformador}

A construção do transformador fora iniciada com as montagens das bobinas de AT (Alta tensão) e BT (Baixa Tensão) e em sequência vem a montagem da parte ativa no tanque junto ao óleo isolante e após os ensaios em laboratório.

Nas Figuras 6 e 7, apresentam-se a montagem da $1^{\mathrm{a}}$ e $2^{\mathrm{a}}$ coluna do transformador.

Na Figura 8 apresenta-se o núcleo já finalizado, com as chapas montadas e toda a parte de prensagem.

Apresenta-se na Figura 9, o transformador finalizado com o comutador de derivação (Tap's) na bobina de AT e com os terminais de BT, após essa montagem fez-se aplicação de verniz em todo o transformador, para melhor compactar as bobinas e ter um ótimo isolamento entre as bobinas.

Depois de realizado todo esse processo a parte ativa foi colocada na estufa para secagem, pois na

$$
E / t=\frac{13800}{12,7}=1087 \text { espiras }
$$

montagem do mesmo foi absorvida umidade no material isolante e também nas soldas realizadas. Este tratamento é realizado em temperaturas de 90 a $100^{\circ} \mathrm{C}$, e deve ter um tempo de secagem com o mínimo de 36 horas segundo procedimento, de acordo com o Manual de Manutenção em Transformadores de Distribuição da P\&L (POWER \& LIGHT ENGENHARIA, 2016), para que o mesmo possa adquirir uma resistência de isolamento nos condutores, evitando assim qualquer risco de curto no seu funcionamento.

Após a secagem na estufa foi feito o fechamento do transformador ao interior do tanque junto com a vedação. E em seguida introduzido o óleo mineral isolante paranífico YPF, que tem a finalidade de refrigerar os enrolamentos e isolamento elétrico. A Figura 10 apresenta a parte ativa no interior do tanque.

Para a colocação do óleo mineral isolante foi utilizada uma bomba chamada de termo vácuo, que tem por finalidade conservar as propriedades isolantes 
Figura 6. $1^{\mathrm{a}}$ e $2^{\mathrm{a}}$ coluna do transformador.

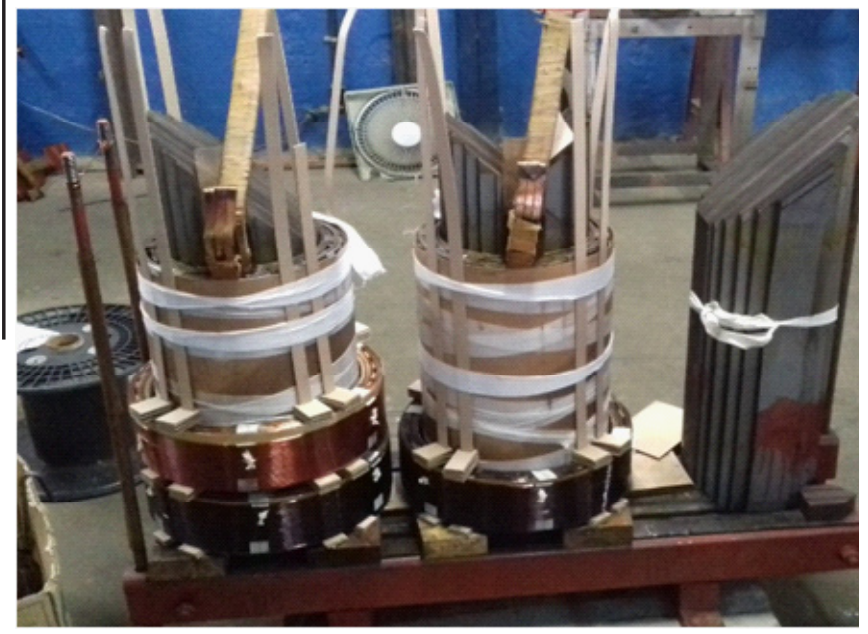

Fonte: Oliveira e Ferreira, 2016.

Figura 8. Montagem das Bobinas Finalizadas.

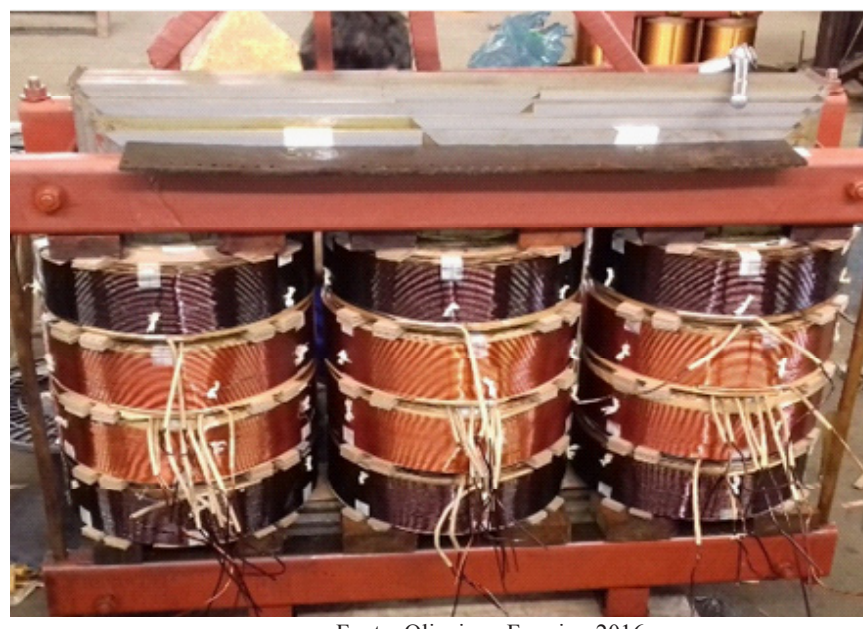

Fonte: Oliveira e Ferreira, 2016.
Figura 7. Montagem das Bobinas Finalizadas.

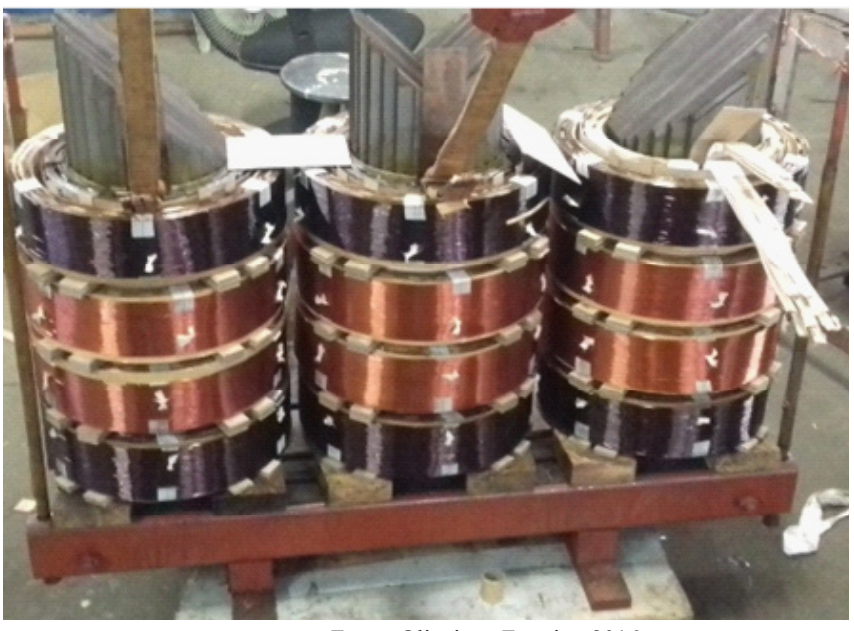

Fonte: Oliveira e Ferreira, 2016

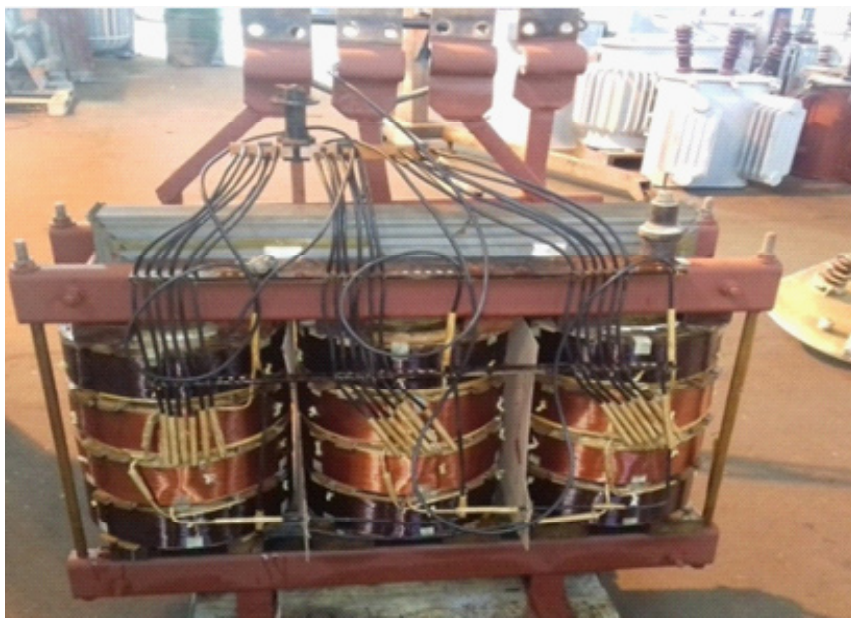

Fonte: Oliveira e Ferreira, 2016 


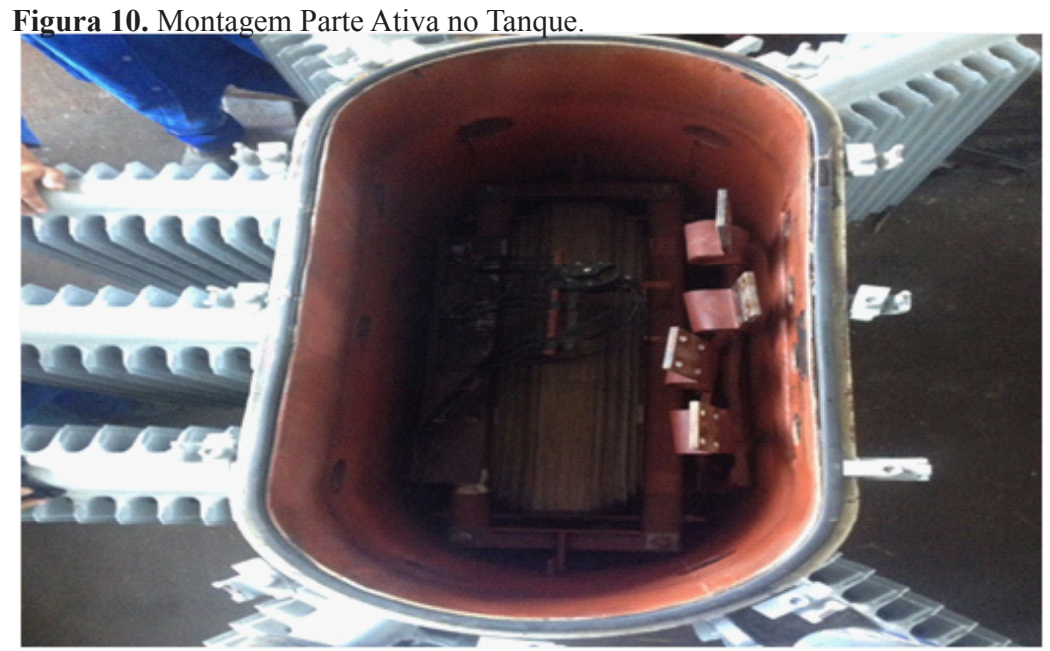

do óleo mineral, não deixando contaminar o óleo com umidade que poderá ocorrer na colocação inadequada garantindo assim uma durabilidade ao transformador. A Figura 11 ilustra a bomba termo vácuo.

Finalizado o fechamento do transformador, com todas as vedações e óleo isolante, levou-se o transformador ao laboratório para realização dos testes e ensaios conforme ABNT NBR 5356:2014.

\section{Ensaios no laboratório}

Os ensaios realizados foram o a vazio e de curto circuito, A Figura 12 ilustra o laboratório e seus instrumentos utilizados no ensaio.

Figura 11. Termo vácuo.

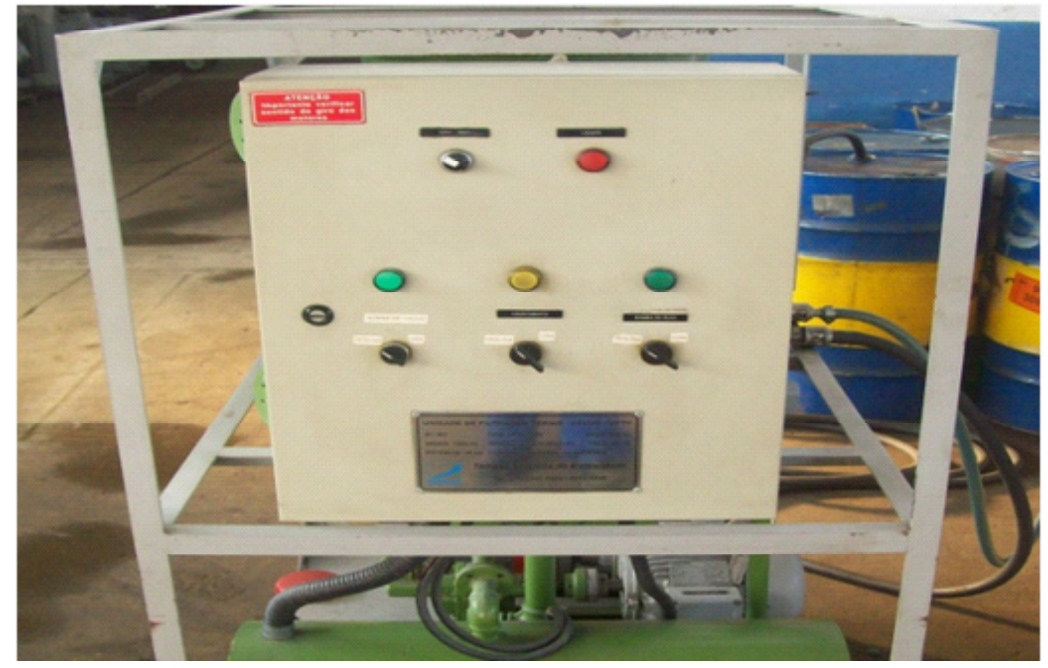

Fonte: Oliveira e Ferreira, 2016.

Figura 12. Mesa e Instrumentos do Laboratório

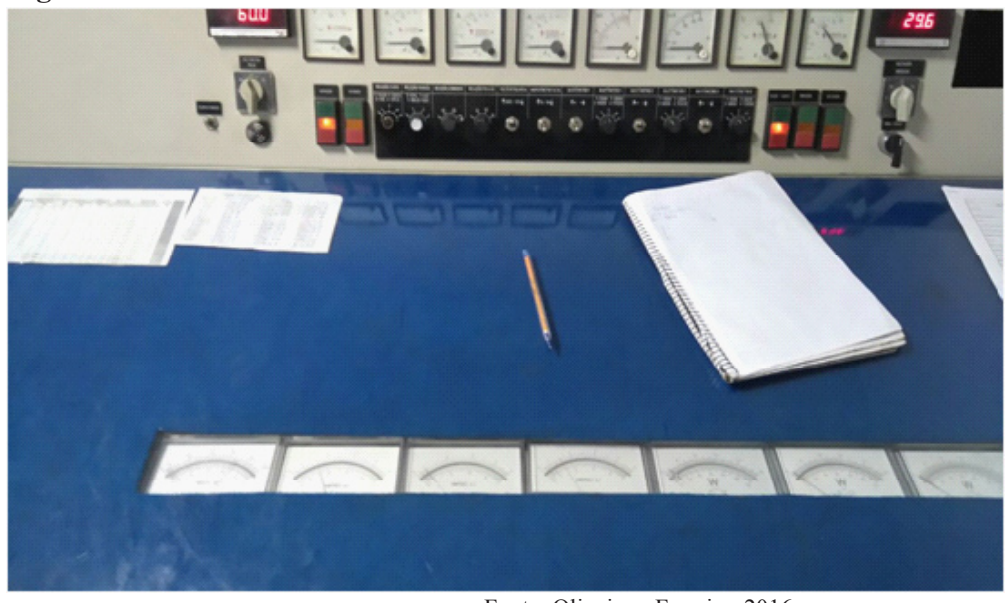

Fonte: Oliveira e Ferreira, 2016 


\section{Ensaio a vazio}

Para este ensaio, verificam-se as perdas no ferro e corrente de magnetização do núcleo.

Dados do transformador:

- Ligação primária- 13.800 volts

- Ligação secundária- 440 volts

Para execução, conectam-se os cabos do laboratório nos terminais de BT do transformador.

Para realizar o ensaio energiza-se o secundário do transformador de acordo com a sua tensão, e mede os valores que serão encontrados nos instrumentos do laboratório.

Utilizando-se os aparelhos para as seguintes funções:

Voltímetro - Realizar leitura da tensão de alimentação do transformador;

Amperímetro - Realizar leitura da corrente das fases; Wattímetro - Realizar leitura da potência dissipada para magnetização do núcleo.

Com a leitura dos instrumentos, obtém-se os valores apresentados no Quadro 2.

Quadro 2. Valores medidos no ensaio a vazio.

\begin{tabular}{|l|l|l|}
\hline Voltímetro & Amperímetro & Wattímetro \\
\hline $\mathrm{V} 1=440$ & $\mathrm{~A} 1=1,5$ & $\mathrm{~W} 1=12$ \\
\hline $\mathrm{V} 2=440$ & $\mathrm{~A} 2=1,0$ & $\mathrm{~W} 2=10$ \\
\hline $\mathrm{V} 3=440$ & $\mathrm{~A} 3=1,5$ & $\mathrm{~W} 3=10$ \\
\hline
\end{tabular}

\section{Análise dos valores encontrados no ensaio a vazio}

Com os valores encontrados no Quadro 2 se calcula o valor da corrente de magnetização ou corrente de excitação e a perda no núcleo. Seguindo-se a Equação 7.

$$
\mathrm{I}^{\prime} 0=\frac{(\mathrm{A} 1+\mathrm{A} 2+\mathrm{A} 3) \times 10}{\mathrm{n} 0 \text { de fases }}
$$

Onde:

I' $0=$ somatório da corrente das 3 fases nas perdas em vazio

10 (escala multiplicativa da mesa)

3 ( $\mathrm{n}^{\mathrm{o}}$ de fases)

Obtendo-se:

$$
\mathrm{I}^{\prime} 0=\frac{(1,5+1,0+1,5) \times 10}{3}=13,33 \mathrm{~A}
$$

Após o cálculo da corrente de excitação, divide-se o somatório da corrente das 3 fases em vazio pela corrente nominal da BT x 100 .

$$
\text { I } 0 \%=(13,3 \div 984) \times 100
$$

$$
\text { I } 0 \%=1,35 \%
$$

Onde:

I $0 \%=$ corrente percentual média ou corrente de excitação

Em um próximo passo obtém-se a soma das potências lidas nos wattímetros nas perdas em vazio (W0).

W0 × 40 (constante multiplicativa proveniente das escalas dos equipamentos do laboratório)

$$
\begin{aligned}
& \mathrm{W} 0=(12+10+10) \times 40 \\
& \mathrm{~W} 0=1280 \mathrm{~W}
\end{aligned}
$$

Com os valores encontrados na corrente de magnetização e nas perdas em vazio, observa-se que os valores encontrados estão dentro dos valores máximos aceitáveis de acordo com a norma ABNT NBR 5440:2014.

\section{Ensaio de curto circuito}

Com a realização deste ensaio verificam-se as perdas nos enrolamentos e o valor da impedância de curto circuito, verificando também se não há curto entre as espiras do enrolamento.

Dado da corrente do primário, obtido na Fórmula 3 deste trabalho $=31,37 \mathrm{~A}$.

Para execução energiza-se somente o primário do transformador, e curto circuito o secundário. A Figura 13 ilustra os cabos conectados aos terminais da AT do transformador e a BT curto circuitada.

Com a leitura dos instrumentos, obtém-se o Quadro 3 que ilustra os valores.

\section{Realizando-se uma análise dos valores encontrados no ensaio de curto circuito}

Com os valores encontrados, calculou-se o valor das perdas totais e a impedância.

\section{Dados:}

I C $\mathrm{C}=$ corrente do curto circuito $=31,37 \mathrm{~A}$

Perdas totais:

$\mathrm{W}^{\prime} \mathrm{CC}=$ soma das potências lidas nos wattímetros, nas perdas do curto circuito $\mathrm{x} 12$

W" C C $=(180+180+180) \times 12=6480 \mathrm{~W}$

Impedância:

$\mathrm{E} Z \%$ = divide a tensão média das perdas em curto circuito pela tensão de AT x100

E Z $\%=(675 \div 13.800) \times 100=4,8 \%$

Impedância parcial $=4,8 \%$

Com os valores encontrados nos ensaios, a vazio e no curto circuito verificou-se que os valores estão de acordo com ABNT NBR 5440:2014, e o transformador terá o rendimento adequado para a carga que irá alimentar. O Quadro 4 apresenta os valores que a norma 
Figura 13. Valores encontrados.

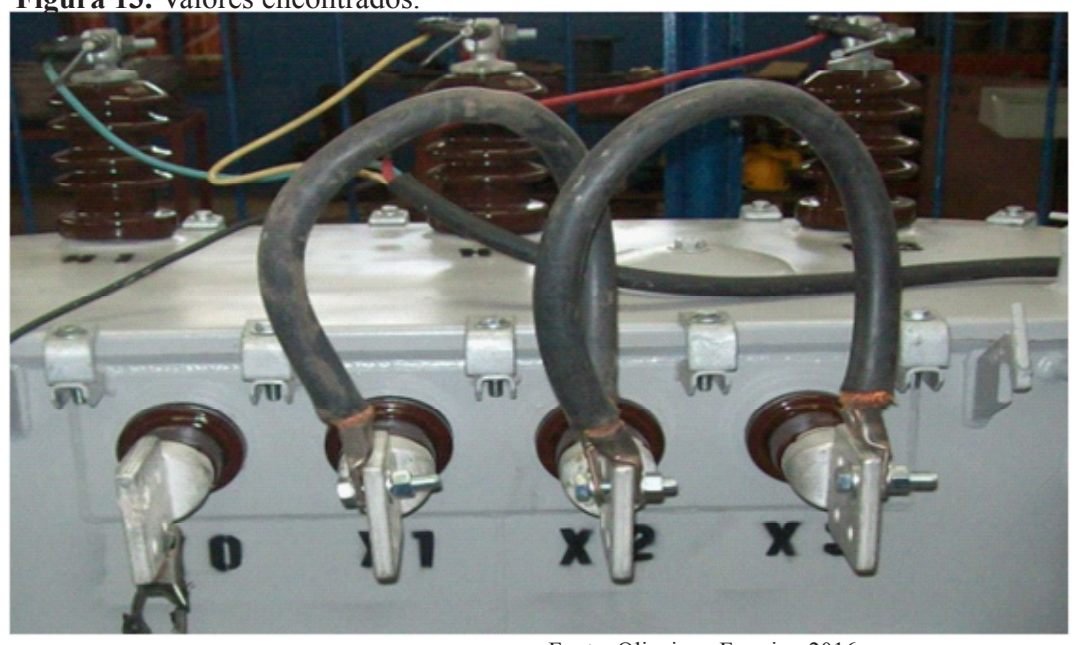

Fonte: Oliveira e Ferreira, 2016.

Quadro 3. Valores encontrados.

\begin{tabular}{|l|l|l|}
\hline Amperímetro & Wattímetro & Voltímetro \\
\hline $\mathrm{A} 1=31,37$ & $\mathrm{~W} 1=180$ & $\mathrm{~V} 1=675$ \\
\hline $\mathrm{A} 2=31,37$ & $\mathrm{~W} 2=180$ & $\mathrm{~V} 2=675$ \\
\hline $\mathrm{A} 3=31,37$ & $\mathrm{~W} 3=180$ & $\mathrm{~V} 3=675$ \\
\hline
\end{tabular}

Fonte: Oliveira e Ferreira, 2016

Quadro 4. Valores orientados por norma.

\begin{tabular}{|l|l|l|l|l|}
\hline & $\begin{array}{c}\text { Perdas } \\
\text { Totais }\end{array}$ & $\begin{array}{c}\text { Perdas em } \\
\text { vazio }\end{array}$ & $\begin{array}{c}\text { Impedância } \\
\text { de Curto } \\
\text { Circuito }\end{array}$ & $\begin{array}{c}\text { Corrente } \\
\text { de } \\
\text { excitação }\end{array}$ \\
\hline 300 & 3360 & 950 & $4,50 \%$ & $2,20 \%$ \\
\hline 500 & 7200 & 1300 & $4,50 \%$ & $1,80 \%$ \\
\hline 750 & 9200 & 1900 & $5 \%$ & $1,70 \%$ \\
\hline
\end{tabular}

Fonte: ABNT NBR 5440:2014.

Quadro 5. Relatório final do transformador.

\begin{tabular}{|c|c|c|c|c|c|c|c|}
\hline F & & $\begin{array}{r}\text { Distrito Ind } \\
\text { CEP: } 27.6 \\
\text { E-m }\end{array}$ & $\begin{array}{l}\text { \&LL TRANS } \\
\text { strial, no - } \\
10-000 \text { i Te } \\
\text { E plengenh }\end{array}$ & $\begin{array}{l}\text { SFORMAD } \\
\text {-65-Parqu } \\
\text { elefax: (24) } \\
\text { taria@pleng }\end{array}$ & $\begin{array}{l}\text { ORES } \\
\text { e Pentagna } \\
\text { 453-7883 i } \\
\text { lenharia.com }\end{array}$ & $\begin{array}{l}\text {-Valença - } \\
2453-6772 \\
\text { abr }\end{array}$ & \\
\hline & ZEL. & ATORIO D & ENSAIO & D EM TRA & NSFORM & AADORES & \\
\hline MARCA: & $P 8$ & & TIPO: & & 80 & N.FABR & 3203 \\
\hline $\begin{array}{l}\text { POTENCLL } \\
\text { TENSAO }\end{array}$ & 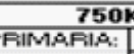 & $\begin{array}{l}\text { KYA } \\
13800 Y\end{array}$ & $\begin{array}{l}\text { FREQU } \\
\text { I PRIMA }\end{array}$ & $\begin{array}{l}\text { JENCIA: } \\
\text { APIO (A): }\end{array}$ & $\begin{array}{l}60 \mathrm{~Hz} \\
31.37 \\
\end{array}$ & $\begin{array}{l}\text { N. FASE: } \\
\text { LIGAC,AO }\end{array}$ & $\begin{array}{c}3 \\
\text { DELTA }\end{array}$ \\
\hline TAP's DOF & PRIMARIO: & $13.800-13$ & $200-12.6$ & $500-12.0$ & $00-11.400$ & & \\
\hline $\begin{array}{l}\text { TENSAO } \\
\text { REG. DE }\end{array}$ & $\begin{array}{l}\text { SECUND: } \\
\text { SERVICO: }\end{array}$ & \begin{tabular}{|l|}
401254 \\
EONTINUC \\
\end{tabular} & $\begin{array}{l}\text { ISECUI } \\
\text { NORMA }\end{array}$ & $\begin{array}{l}\text { ND. }(A): \\
\text { A ABNT: }\end{array}$ & \begin{tabular}{|c|}
984 \\
NBR 5441 \\
\end{tabular} & $\begin{array}{l}\text { LIGACCAOO } \\
\text { RESFR: }\end{array}$ & $\begin{array}{c}\text { ESTAELA } \\
\text { ONAN }\end{array}$ \\
\hline & & RELA & AO DE & IRANSFC & PAMACAO & & \\
\hline TENSAO: & 13.800 & 13.200 & 12.600 & 12.000 & 11.400 & & \\
\hline FASE B & 54.330 & 51,968 & 49.606 & 47.244 & 44.881 & & \\
\hline FASES & 54.336 & 51,965 & 49.609 & 47.250 & 44.886 & & \\
\hline FASEI & 54.329 & 51,966 & 49.610 & 47.246 & 44.890 & & \\
\hline RESIST & ENCIA DE & ENROL & 3 & RESIS & TENCIA D & E ISOLAR & IENTO $(\mathrm{M} \Omega)$ \\
\hline & TEMPEF & ATURA: & & AT & $\mathrm{IBT}$ & & 000.00 \\
\hline ALTATE & $\operatorname{VSAO}[\Omega]$ & BAIXA TER & $\mathrm{AO}(\mathrm{m} \Omega)$ & AT IN & AASSA & & 000,00 \\
\hline FASEB & 10.00 & FASE B & 22,60 & BT i & AASSA & & 000,00 \\
\hline FASES & 10.00 & FASES & 22,50 & MEGOHI & METRO DE & & $000 \mathrm{~V}$ \\
\hline FASE I & 10.00 & FASE I & 22.70 & TEMPE & RATURA & & $6,0^{\circ} \mathrm{C}$ \\
\hline & PER & DAS & & EORAEN & TE DE EXI & CITACAG & TENSAO DE \\
\hline EMV & Q.ZIO: & 1280 & & & & & EXPEDICAO \\
\hline $\begin{array}{r}\text { EMC.O } \\
\text { TOT }\end{array}$ & $\begin{array}{l}\left(75^{\circ} \mathrm{C}\right) \text { : } \\
\text { A.IS: }\end{array}$ & $\frac{6481}{7760}$ & & & $1.35 \%$ & & \\
\hline RENDI & MENTO A & PLENA C & RGA & RE & GULAGEM & A PLENS & CARGA \\
\hline POLAF & IDADE: & SUBTR & TIYA & SEQU & NCIADEF & ASES: & $\mathbf{A}-\mathbf{S}-\mathbf{T}$ \\
\hline DESLOC. & NGULAR: & 30 & & IMPE & DANCIA A & $75^{\circ} \mathrm{C}:$ & $4.80 \%$ \\
\hline RIGIDEZ DI & ELETRICA: & $40 K$ & & TEN: & SAODE ENS & 5.A.IO: & $13800 \mathrm{Y}$ \\
\hline QUANT. & LIQUIDOIS & OLANTE: & $650 \mathrm{~L}$ & PESO & TOTAL: & & $620 \mathrm{~kg}$ \\
\hline PESO & DAPARTE & ATIVA: & & DATAD & DENSAIO: & & 0312016 \\
\hline
\end{tabular}


orienta, todos os valores encontrados não ultrapassaram os valores orientados por norma.

\section{Relatório final}

Após todos os testes e ensaios realizados obtémse o relatório final do transformador ensaiado conforme apresentado no Quadro 5.

\section{Placa de Identificação}

Encerrados os testes e aprovações, criou-se a placa de identificação do transformador conforme apresentado na Figura 14.

Figura 14. Placa de Identificação.

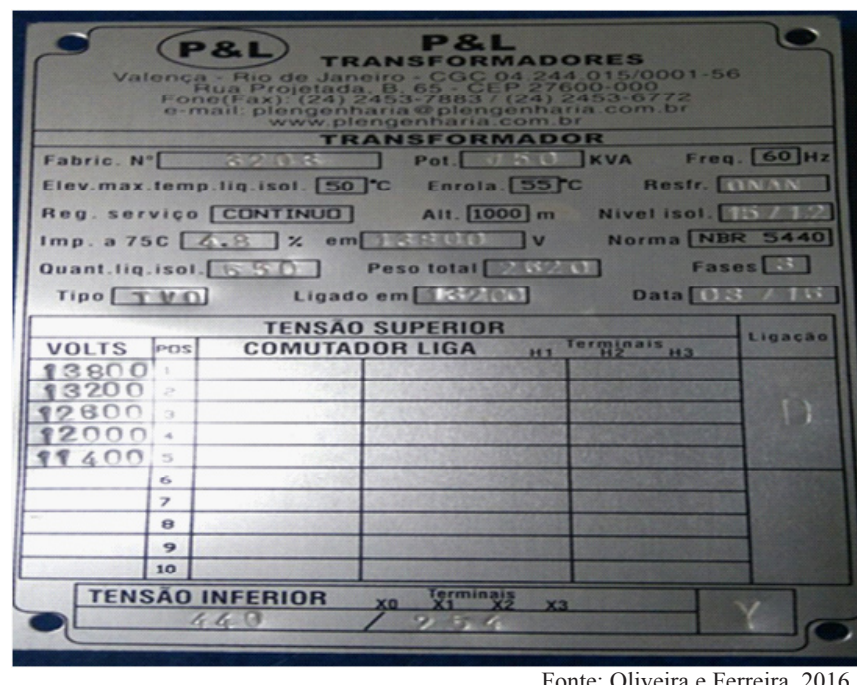

\section{Conclusão}

Após a elaboração deste projeto, montagem e ensaios finais o transformador terá um bom desempenho de funcionamento, pois os resultados encontrados nos ensaios em laboratório estão de acordo com as normas exigidas pela ABNT- NBR 5440:2014 e ABNT NBR 5356:2014.

Obteve-se uma repotenciação com grande aumento de potência, pois, atingiram-se os limites máximos de espaçamentos exigidos por normas, pois não se permite passar dos limites de espaçamentos que as normas orientam, sejam entre as bobinas ou referentes ao espaçamento com da parte ativa ao tanque, o que acarretaria uma vida útil muito pequena ao equipamento, onde o mesmo sofreria com aquecimento excessivo e não atendendo a sua carga desejada.

Os resultados obtidos apresentam um grande incremento de potência, baseando-se nos resultados encontradosnonovoprojetoimplantadoaotransformador que possuía uma potência nominal de $650 \mathrm{kVA}$, e com a repotenciação verificou-se que se obteve um aumento na sua potência nominal em 15,39\% aproximadamente (100 kVA), pode-se afirmar que tais resultados são expressivos.
Pode-se então apontar como grande vantagem obtida neste projeto de repotenciação onde o núcleo do transformador operava com potência nominal de 650kVA (com entrada de $13.8 \mathrm{kV}$ em delta tendo 5 tap's de derivação e sua saída em 440/254 volts em estrela), que após todas as etapas realizadas o mesmo passou a operar com potência de $750 \mathrm{kVA}$, com as mesmas características que possuía anteriormente, obedecendo todas as normas vigentes com valores normais para operação, sem qualquer elevação de temperatura.

Com os dados obtidos após repotenciação do transformador pode-se afirmar que o objetivo desse artigo de apresentar as técnicas de aumento de potência nos transformadores antigos, das redes de distribuição, aproveitando-se do espaçamento da janela do núcleo obedecendo-se ao isolamento entre as bobinas, foi alcançado com sucesso.

Não se apresentam desvantagens no projeto, trata-se de uma repotenciação e o ganho obtido na potência do transformador é válido. Vale ressaltar que todas as etapas de repotenciação realizadas seguiram as normas e todos os procedimentos necessários para obtenção de resultados satisfatórios para uma melhor repotenciação.

\section{Referências}

ALMEIDA, Antônio Tadeu Lyrio. Operação e Manutenção em Transformadores. Escola Federal de Engenharia de Itajuba. 2000.

ASSOCIAÇÃO BRASILEIRA DE NORMAS TÉCNICAS - ABNT. NBR 5356 - Transformador de potência. Rio de Janeiro, 2014

NBR 5440 - Transformadores para redes aéreas de distribuição Padronização. Rio de Janeiro, 2014.

HALLIDAY, David, RESNIK Robert, KRANE, Denneth S. Física 3, volume 2, 5 Ed. Rio de Janeiro: LTC, 2004. 384 p.

LORENCINI BRASIL. Soluções em Análise de Óleo. Artigo. Disponível em: $<$

$<$ http://www.lorencinibrasil.com.br/blog/equipamentos-associadostransformadores-buchas-e-comutadores/> Acesso em fevereiro de 2016.

MARTIGNONI, Afonso. Transformadores. São Paulo, $8^{\mathrm{a}}$ Edição, Editora Globo, 1991

OLIVEIRA, Guilherme Ferraz de; FERREIRA, Alex Franco. Repotenciação de um Transformador de Potência Trifásico de 650 kVA / 750 kVA em Proveito do Aumento de Potência. 2016. 92 f. TCC (Graduação) - Curso de Engenharia Elétrica, Ciências Tecnológicas e Sociais Aplicadas, Universidade Severino Sombra, Vassouras, 2016.

O Setor Elétrico Edição 38, março de 2009. Disponível em: <http://www. osetoreletrico.com.br/web/component/content/article/58-artigos-e-materiasrelacionadas/89-tcc-reprojeto-de-transformador-com-aumento-de-potencia. html>. Acesso em 15 de fevereiro de 2016.

POWER \& LIGHT ENGENHARIA. Transformadores - Manual de manutenção em transformadores de distribuição. Disponível em: $<\mathrm{http}$ :// www.plengenharia.com.br/index.php> Acesso em 25 de fevereiro de 2016.

SANTOS, Marco Aurélio Da Silva. "A Lei de Lenz"; Brasil Escola. Disponível em $<$ http://brasilescola.uol.com.br/fisica/a-lei-lenz.htm>. Acesso em 06 de abril de 2016.

WEG INDÚSTRIAS S.A. - Transformadores. Informações Técnicas DT-11. Características e Especificações de Transformadores de Distribuição e Força, Disponível em: <www.weg.com.br> Acesso em fevereiro de 2016. 